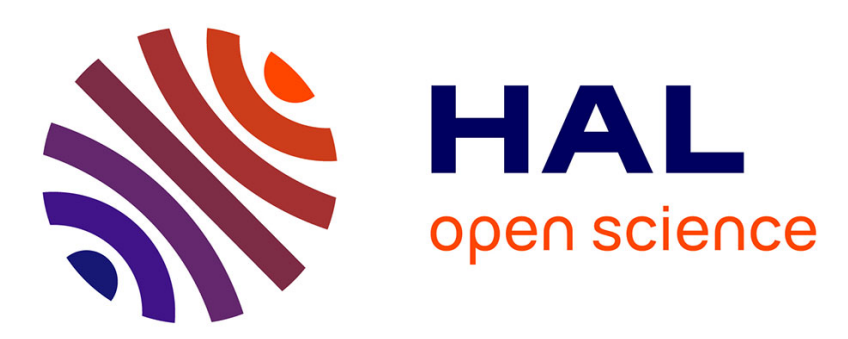

\title{
SOFT CHEMICAL STRIPPING OF ALUMINIDE COATINGS AND OXIDE PRODUCTS ON NI SUPERALLOYS
}

S. Poupard, J.F Martinez, Fernando Pedraza

\section{- To cite this version:}

S. Poupard, J.F Martinez, Fernando Pedraza. SOFT CHEMICAL STRIPPING OF ALUMINIDE COATINGS AND OXIDE PRODUCTS ON NI SUPERALLOYS. Surface and Coatings Technology, 2008, 202, pp.3100-3108. 10.1016/j.surfcoat.2007.11.013 . hal-00512240

HAL Id: hal-00512240

https://hal.science/hal-00512240

Submitted on 28 Aug 2010

HAL is a multi-disciplinary open access archive for the deposit and dissemination of scientific research documents, whether they are published or not. The documents may come from teaching and research institutions in France or abroad, or from public or private research centers.
L'archive ouverte pluridisciplinaire HAL, est destinée au dépôt et à la diffusion de documents scientifiques de niveau recherche, publiés ou non, émanant des établissements d'enseignement et de recherche français ou étrangers, des laboratoires publics ou privés. 


\title{
SOFT CHEMICAL STRIPPING OF ALUMINIDE COATINGS AND OXIDE PRODUCTS ON NI SUPERALLOYS
}

S. Poupard, J-F. Martinez, F. Pedraza*

Université de la Rochelle. Laboratoire d'Etudes des Matériaux en Milieux Agressifs (LEMMA -EA 3167). Bâtiment Marie Curie, Avenue Michel Crépeau, F-17042 La Rochelle cedex 01, France.

* Corresponding author: F. Pedraza

Tel. : +33(0)546458297. Fax : +33(0)5.46457272.e-mail address : fpedraza@univ-lr.fr

\begin{abstract}
Turbine components are submitted to very aggressive environments and particularly to high temperature corrosion and /or oxidation. Because of their high cost, they have to be repaired instead of being replaced. Prior to refurbishment and recoating, the components have to be fully stripped to remove the oxide products and defective coatings.
\end{abstract}

In this work, various soft chemical stripping methods are studied based on pitting acids and agents as well as oxidising acids in organic and aqueous media. The effect of the immersion time is also investigated. The feasibility of the dissolution reactions has been evaluated through thermodynamic calculations. It will be shown that the most effective baths are those containing relatively high concentrations of pitting reagents in organic media. Aqueous solutions lead to pitting of the superalloy. The stripping mechanisms have been discussed in terms of the dissolution of coating grain boundaries once the solution has passed through the oxide scales.

Keywords: soft chemical stripping, aluminide coating, oxide scales, Ni-base superalloy.

\section{1.- Introduction}

During the last decades, the temperature of gas turbine engines has been increased in order to improve the combustion efficiency. As a consequence, most of hot sections components (blades, vanes...) of industrial and aero turbines are coated to provide protection from oxidation and/or corrosion attacks by developing a protective aluminium oxide scale [1]. During their exposure to high temperatures and under high pressure, these external coatings are damaged. Therefore, the entire coatings need to be stripped prior to refurbishment and 
recoating operations of the turbine components. Nowadays, many techniques exist to strip aluminide coatings. Mechanical processes can be classified as abrasive methods that have a scouring action. The impact methods are based on particle impact to crack the coating. Due to the differences in hardness or brittleness, control of the system and performing the stripping without damaging the substrate remains problematic [2]. Chemical stripping generally involves the use of strong inorganic acids that may reduce the substrates cross-section, hence compromising the mechanical properties of the component. For the specific case of aluminide coatings, Amirkhanova and Nev'yantseva [3] employed a mixture of nitric and hydrochloric acids $(2: 1)$ to which potassium dichromate and ammonium heptamolybdate were added to increase the oxidising power. Nevertheless, the presence of $\mathrm{NH}_{4}{ }^{+}, \mathrm{Mo}_{7} \mathrm{O}_{24}{ }^{6-}$ and $\mathrm{Cr}_{2} \mathrm{O}_{7}{ }^{2-}$ ions represents non negligible environmental hazards [4]. Some patented approaches employ very complex acidic mixtures comprising an organic sulfonic acid, inorganic acids, and other, complexing, oxidising and reducing agents to remove the aluminide coating [5]. The hot corrosion products can be previously removed using heated solutions of acetic acid [6]. These results have been supported in recent works $[7,8]$ at other elevated temperatures and with the addition of an oxidising agent. Furthermore, chemical stripping typically requires previous surface preparation steps (e.g. sand or water jet blasting, vapour degreasing etc.) that make the overall process expensive and time consuming. This study will therefore focus on alternative "soft" chemical stripping approaches capable of stripping aluminides and their oxides without damaging the substrate in a single step process. The reactivity of various solutions against an untreated, an aluminide coated and a coated and oxidised superalloy substrate will be studied from theoretical and experimental approaches to highlight the likely mechanisms involved in soft chemical stripping.

\section{2.- Experimental procedure}

\section{1.- Materials of study}

SIFCO Turbine Components provided a nickel based superalloy (Cannon Muskegon) in the form of bars of $12.5 \mathrm{~mm}$ of diameter from which different $2 \mathrm{~mm}$-thick disks were cut by electrodischarge machining (EDM). The nominal composition is given in Table 1. Some coupons were also CVD-aluminised at $\mathrm{SIFCO}$ at $1050^{\circ} \mathrm{C} / 6 \mathrm{~h}$. Prior to aluminisation, a typical SIFCO preparation procedure including sand blasting and vapour degreasing was followed. The aluminised samples were subjected to cyclic oxidation tests in a tubular furnace (Carbolyte Type 201) during 10 cycles and atmospheric pressure of air. Each cycle was consisted in 24 hours at $1100^{\circ} \mathrm{C}$ and a rapid air cooling to room temperature for $15 \mathrm{~min}$. 
Between the cycles, the specimen mass changes were recorded using a $10^{-5} \mathrm{~g}$ accuracy Precisa XR205 SR-DR balance.

\section{2.- Experimental Set-up}

The experimental set-up employed for the stripping tests is composed of a $250 \mathrm{~mL}$ beaker containing the stripping solution, a Teflon coated probe CheckTemp CKT2 thermometer to control the temperature with a $\pm 0.3^{\circ} \mathrm{C}$ accuracy at $50^{\circ} \mathrm{C}$, a hot plate stirrer (Heidolph 3002) to heat the solution while homogenising the composition of the solution.

Thermodynamic calculations have been performed in order to study the theoretical feasibility of the chemical reactions between the stripping solution and the substrates of concern. For such purpose, the HSC Chemistry thermodynamic software [9] has been employed. The different species, their physical states, their initial amounts, pressure (1 bar) and different temperatures are entered and linked to the database existing in the software.

The composition of the stripping solutions is gathered in Table 2. Because of confidentiality, the reagents cannot be fully specified. All the baths are strongly acidic $(\mathrm{pH}<1)$. The experimental temperature was fixed at $50^{\circ} \mathrm{C}$ since a previous study had shown that the best stripping yield was obtained at this temperature [8]. The organic medium was chosen from the family of aliphatic compounds having a high evaporation temperature. No degradation of the organic medium at the stripping temperature occurred as verified by infrared spectroscopy in a Thermo Nicolet FT-IR Nexus spectrometer. The immersion times were set at $30 \mathrm{~min}, 1,2$, and 4 hours in order to follow the evolution of the stripping process. Before and after immersion in the stripping baths, the samples were cleaned in isopropyl alcohol using ultrasonic baths and subsequently dried with hot air. They were then weighed in a $10^{-4} \mathrm{~g}$ accurate Mettler Toledo B154 balance in order to estimate the loss in material thickness.

\section{3.- Characterisation techniques}

X-ray diffraction measurements were carried out with a diffractometer Bruker AXS D8 Advance using $\mathrm{CuK} \alpha$ radiation $(0.15406 \mathrm{~nm})$ in the $\theta-2 \theta$ configuration. An optical microscope Olympus CK40M was also employed to evaluate the surfaces and the cross sections and to estimate the stripped thickness. Scanning electron microscopy was performed in a JEOL 5410LV coupled to a Rontec detector to perform Energy Dispersive Spectrometry (EDS) analyses. For the metallographic preparation of the cross sections without damaging 
the different surface layers, thin copper layers were electrodeposited on the oxidised specimens [10]. Accurate quantification of the four main elements ( $\mathrm{Al}, \mathrm{Ni}, \mathrm{Co}$ and $\mathrm{Cr}$ ) from the coating being dissolved was determined by inductively coupled plasma-optical emission spectroscopy (ICP/OES) in a Varian Vista-Pro operating between 167-785 nm.

\section{3.- Results and discussions}

\section{1.- Materials of study}

The morphology and composition of the CVD aluminide coating is given in Figures 1 (a) and (b), respectively. It can be observed that the coating can be divided into two main sections. The outermost band (additive layer) is about $30 \mu \mathrm{m}$ thick and has a very homogeneous morphology. The Al and Ni contents revealed by EDS microanalyses suggest the major formation of a NiAl phase in this area, which is confirmed by XRD. Beneath the additive layer, the interdiffusion layer appears as a result of the countercurrent diffusion of $\mathrm{Al}$ being transported inwardly and the substrate elements diffusing outwardly [8,11]. A $30 \mu \mathrm{m}$ thick layer is then developed. The Al content in this zone drops drastically compared to the additive layer and the $\mathrm{Al} / \mathrm{Ni}$ ratios suggest that $\mathrm{Ni}_{3} \mathrm{Al}$ may be the major component of the matrix. Many of the white precipitates close to the substrate contain significant amounts of solid solution strengtheners (Ta, W, Hf, etc.) and can be unambiguously be ascribed to topologically closed-packed phases (TCP) [11].

Cyclic oxidation tests have been carried out on the coated materials in order to simulate real conditions in service, thus bringing about scale cracking, spallation of the oxide scales and inducing strong evolutions in the composition and morphology of the underlying coatings (interdiffusion) [12-14]. To this end, two types of aluminide coatings were tested and the evolution of the mass gain per surface unit as a function of the number of cycles was recorded. For the Al-poor coatings ([Al] $<<50$ at $\%$ at the topmost coating), significant mass losses were observed. This was related to extensive spallation of scales and to the development of poorly protective oxides like the blue-green $\mathrm{NiAl}_{2} \mathrm{O}_{4}$ spinel oxide [15]. Conversely, in the Al-rich coatings ([Al] $>50$ at $\%$ at the topmost coating) well adhered oxide scales were developed, hence impeding the formation of non protective oxides and their subsequent spallation [16]. The scales mainly developed on top of the original NiAl grains.

The XRD analyses of the coated substrates after the overall $240 \mathrm{~h}$ of oxidation are shown in Figure 2. Both the Al-poor and the Al-rich coatings develop the protective $\alpha-\mathrm{Al}_{2} \mathrm{O}_{3}$ alumina, 
as well as the less protective $\mathrm{NiAl}_{2} \mathrm{O}_{4}$ spinel oxide. The appearance of the $\mathrm{Ni}_{3} \mathrm{Al}$ phase indicates that the $\mathrm{NiAl} \rightarrow \mathrm{Ni}_{3} \mathrm{Al}$ transformation has occurred in the coating as a result of $\mathrm{Al}$ depletion and Ni enrichment [17]. Further comparison of the intensity ratios of the main peaks $\mathrm{Al}_{2} \mathrm{O}_{3}$ (104), $\mathrm{NiAl}_{2} \mathrm{O}_{4}$ (311) and $\mathrm{Ni}_{3} \mathrm{Al}$ (111) between the Al-poor and the Al-rich coatings indicates that a thicker oxide scale is present on the Al-rich coating surface. Moreover, the Alpoor coatings seem to develop a higher content of spinel oxides after $240 \mathrm{~h}$ of oxidation. This can be explained by the continuous spallation of the oxide layer occurring from $72 \mathrm{~h}$ of oxidation for the Al-poor coatings and could indicate that aluminium transport towards the surface is not sufficient to reform the alumina scale thus inducing the transformation $\mathrm{NiAl} \rightarrow$ $\mathrm{Ni}_{3} \mathrm{Al}[17]$.

In both cases, brighter areas appear under the oxide scale and correspond to $\mathrm{Al}$ depletion, thus inducing the $\mathrm{NiAl} \rightarrow \mathrm{Ni}_{3} \mathrm{Al}$ transformation as also identified by XRD.

The SEM cross sections morphologies of the Al-poor coatings after $240 \mathrm{~h}$ of cyclic oxidation is shown in Figure 3(a). It can be observed that the oxide scale is continuous close to the substrate and partially discontinuous at the coating/gas interface as a result of spallation. According to the EDS analyses, the scale is mainly composed of $\mathrm{Al}$ and $\mathrm{O}$ and agrees with the XRD results (Figure 2). Comparatively, the Al-rich coatings [Figure 3(b)] exhibit a continuous scale of $\mathrm{Al}_{2} \mathrm{O}_{3}$ interrupted by nodules. The composition of nodules indicates mainly the presence of $\mathrm{Al}$ and $\mathrm{O}$ with $\mathrm{Ni}$ enrichment at the base of the nodules, which agrees well with similar results observed in the case of stainless steels [18]. In both types of coatings, brighter areas appear in the backscattered electron mode. These are mainly located under the oxide scale and in the interdiffusion layer and correspond to Al depletion, thus inducing the $\mathrm{NiAl} \rightarrow \mathrm{Ni}_{3} \mathrm{Al}$ transformation as also identified by XRD. The extent of the transformation is more significant in the Al poor-coatings than in the Al-rich ones.

\section{2.- Stripping tests on uncoated samples}

The experimental approach has been realised by gravimetric studies in order to estimate the efficiency of each stripping procedure. To this end, the calculations have been done considering the samples as perfect cylinders of radius $R$ and initial and final thickness $e_{i}$ and $e_{f}$, respectively. Then, the initial volume of the sample is $V_{i}=\pi \cdot R^{2} \cdot e_{i}$ and the final one is $V_{i}=$ $\pi \cdot R^{2}$. $e_{f}$. If we consider the same surface before and after stripping $\left(S_{f}=S_{i}\right)$ and the density 
(calculated from a sample) equal to $\rho=\mathrm{m}_{\mathrm{i}} / \mathrm{V}_{\mathrm{i}}=\mathrm{m}_{\mathrm{f}} / \mathrm{V}_{\mathrm{f}}=8.64 \mathrm{~g} / \mathrm{cm}^{3}$, the thickness of the dissolved material is given by the relation $<1>$ :

$$
\mathrm{e}_{\mathrm{i}}-\mathrm{e}_{\mathrm{f}}=\frac{\mathrm{m}_{\mathrm{i}}-\mathrm{m}_{\mathrm{f}}}{\rho \cdot \Pi \cdot \mathrm{R}^{2}}
$$

The comparison of the surfaces of the raw substrate after immersion in baths A (pitting acidorganic media) and A1 (pitting acid-aqueous media) seems to indicate that the pitting acid is far more aggressive in water than in organic media. Moreover, the SEM observations (not shown) have revealed pitting of the surface when employing aqueous solutions. Actually, the $\mathrm{O}-\mathrm{H}$ bond in an alcohol (R-OH) is polarised by the electronegativity of $\mathrm{O}$ but not as much as in water because of the inductive effect of the organic group (R-). The action of a strong protonic acid leads to $\mathrm{R}-\mathrm{O}^{+}-\mathrm{H}_{2}$ by the fixation of $\mathrm{H}^{+}$on the free doublet of oxygen. In acid media, C-O rupture occurs, which reduce the solution reactivity [19]. Therefore, only the tests in organic media are discussed in the following.

The evolution of thickness loss with immersion time is depicted in Figure 4. It can be observed that the main thickness loss occurs with solution A2 because of the higher content of pitting reagents in this solution, in agreement with the HSC [9] thermodynamic calculations. Furthermore, the surface is rather homogeneous and remains basically free of pits, which could be promising in the perspective of stripping the aluminide coatings. However, dissolution occurs very rapidly and surpasses the acceptable limit typically agreed to avoid significant reduction of the cross section of the components [7]. For the remaining solutions (A, B and C), bath B shows almost no activity against the Ni superalloy as the oxidising acid could passivate the surface thus impeding active dissolution [20,21]. Besides, microscopy observations reveal non homogeneous surfaces with some preferential dissolution. This can be probably due to partitioning of the alloying elements to the $\gamma$ and $\gamma^{\prime}$ phases, hence to different reactivity. Solutions A and $\mathrm{C}$ seem to dissolve similarly the uppermost layers of the substrate for the shortest immersion times. After $4 \mathrm{~h}$ of stripping, the thickness loss is twice using bath $\mathrm{C}$ compared to bath $\mathrm{A}$ indicating that a combined effect of pitting and oxidising acids could effectively strip the superalloy substrate.

\section{3.- Stripping trials on aluminised substrates}

The reactivity of the aluminide coatings against the different baths in organic medium has been first evaluated by calculating the free energy of reaction $\left(\Delta \mathrm{G}^{\circ} \mathrm{r}\right)$ at $50^{\circ} \mathrm{C}$ for the reaction 
model $<1>$, where $\mathrm{RH}$ is the reagent, $\mathrm{O}$ the organic solvent and $\mathrm{R}$ - the product anion and O-R the. All the baths lead to spontaneous dissolution reactions, especially in bath A2, where the pitting reagent concentration is the highest.

Figure 5 shows the evolution of the X-ray patterns of the aluminide coatings after different immersion times in bath A (Pitting acid- Organic media). It can be readily seen that after 30 min of stripping the NiAl peaks of the as-deposited coating start vanishing and a new major peak diffracting at about $50^{\circ}$ appears. This can be unambiguously ascribed to the (200) reflection of the raw substrate. Some other small peaks appear and are related to carbides and TCP phases [8]. This is in agreement with the SEM observations (Figure 6) in which the interdiffusion layer is not completely dissolved after $1 \mathrm{~h}$. Conversely, after $2 \mathrm{~h}$ of immersion, the coatings seem to be fully removed and unpitted and very flat surfaces appear, especially after $4 \mathrm{~h}$ of immersion. The X-ray peak appearing at about $48^{\circ}$ after $4 \mathrm{~h}$ of stripping could correspond to the (200) planes from a martensite originating after the quenching step of the coating process [22].

Concerning the bath B (Oxidising acid-Organic media) no macroscopic effect on the removal of the aluminide coating has been observed. After $4 \mathrm{~h}$ of stripping, the sample has kept its original aspect. Moreover, the XRD patterns are similar to the XRD diffractogramme of an as-coated sample, thus confirming the inefficiency of bath B. Despite the thermodynamic spontaneity of the reaction, it is believed that passivation of the surface is the major mechanism occurring in this medium which impedes dissolution of the aluminide coating $[20,21]$. Therefore, this single approach is not retained for further stripping tests.

According to the thermodynamic calculations, a mixture of both a pitting and an oxidising acid (bath $\mathrm{C}$ ) could be energetically able to dissolve NiAl. The X-ray diffraction analyses shown in Figure 7 indicate the progressive disappearance of the NiAl peaks of the coating with immersion time. After 1 hour, the bath seems to dissolve the additive layer but not the interdiffusion layer. The coating is completely dissolved after $4 \mathrm{~h}$ of immersion as the characteristic peak (200) of the substrate appears. These results are rather in agreement with the observations of the cross sections by SEM, which are in turn similar to those presented in Figure 6. After $2 \mathrm{~h}$ of stripping, the remaining TCP phases and carbides at the interdiffusion layer/substrate interface makes the surface more heterogeneous but these could be dissolved upon further repair steps involving heat treatments. 
As for bath A2 (Pitting acid-Pitting agent-Organic media), the thermodynamic calculations have also shown the possibility to dissolve NiAl. The X-ray diffraction patterns (Figure 8) show that $4 \mathrm{~h}$ of immersion in bath A2 are in fact required to achieve the complete removal of the aluminide coating. This is in agreement with the SEM study of the cross sections but in contrast with the significant reactivity of the uncoated substrate towards this solution. This suggests that the dissolution action is probably tempered by the fixation of the anion of the pitting reagents to the polarized organic molecule [19] as if the solubility product had been achieved. The small concentration of free pitting reagents would then dissolve the coating. Therefore, longer immersion times would be required.

\section{4.- Stripping trials on oxidised coated substrates}

After $4 \mathrm{~h}$ of stripping, the samples have been systematically analysed by XRD as shown in Figure 9. For the Al-poor coatings after oxidation, the XRD patterns show the characteristic peaks of $\mathrm{Ni}_{3} \mathrm{Al}$ and $\mathrm{Al}_{2} \mathrm{O}_{3}$ regardless of the bath employed. The spinel $\mathrm{NiAl}_{2} \mathrm{O}_{4}$ seems to be present after stripping in solutions $\mathrm{A}$ and $\mathrm{C}$ but it has totally disappeared with bath $\mathrm{A} 2$. For the Al-rich coatings, baths A and A2 seem to strip effectively the coatings and their oxides as shown by the appearance of the characteristic peak of the substrate. Nevertheless, some peaks of $\mathrm{Ni}_{3} \mathrm{Al}$ remain with a more important intensity for bath $\mathrm{A}$, together with some carbides and TCP phases. Conversely, in bath C, the presence of the (200) peak slightly shifted towards higher angles as well as the one reflecting at about $75^{\circ}$ may indicate a significant contribution from $\mathrm{Ni}_{3} \mathrm{Al}$.

The SEM surface and cross section morphologies of the oxidised Al-poor specimens have shown that the oxide scales have not been fully removed with any of the 3 solutions and that the coatings have been therefore only partly stripped. This could indicate that the oxide scale does not contain enough cracks that allow permeation of the stripping solution. Furthermore, the presence of the spinel oxides detected by XRD could also hinder the penetration of the solution. Indeed, it has been shown that this oxide normally appears at the top of the original grain boundaries of the coating [15]. Therefore, sand blasting operations prior to stripping are recommended in the case of Al-poor coatings.

Conversely, the oxidised Al-rich coated samples seem to be more dissolved as shown in Figure 10. Baths A and $\mathrm{C}$ are able to strip most of the coating after $4 \mathrm{~h}$ of immersion but some 
isolated islands of the oxides that had grown over the columnar NiAl grains of the coating still remain. The EDS analyses of different areas show that these nodules are basically composed of aluminium and oxygen, whereas the small grains underneath are rich in refractory elements and the spongy structure is rich in Ni. These observations agree with the XRD patterns of Figure 9(b), where alumina, TCP phases and Ni derivatives had been detected. The solution A2 [Figure 10(b)] dissolves further the superficial layers than baths A and C. The surface is relatively homogeneous despite the presence of small oxide nodules and some pitting. The EDS microanalyses indicate the basic composition of the superalloy.

\section{4.- Dissolution mechanisms}

According to the previous results, it seems that bath A (Pitting acid-Organic media) would present the faster dissolution kinetics. On the contrary, in bath B (Oxidising acid-Organic media) the anions do not seem to have a significant effect on the dissolution of $\beta$-NiAl phases. Therefore, the pitting anionic species could play a major role. This is clearly reflected in Figure 11, where the concentration of the major constituents of the coatings ( $\mathrm{Ni}, \mathrm{Al}, \mathrm{Co}$ and $\mathrm{Cr}$ ) is plotted against immersion time for baths $\mathrm{A}$ and $\mathrm{C}$. The chosen metals are well known for their high sensitivity to pitting media. Therefore, the more the solution is concentrated in pitting anionic species; the more the coating thickness should be dissolved. This is the case of solution A, where the amount of dissolved cation increases with increasing time in linear fashion. As more nickel is dissolved, the solutions are become greener and greener. Conversely, the concentration of species dissolved in bath $\mathrm{C}$ (Pitting acid-Oxidising acidOrganic media) does not follow a linear law [Figure 11(b)], probably because a conversion layer is formed on the outermost surface hence impeding subsequent dissolution of the metallic phases [20,21]. In this sense, solution A2 should give the fastest dissolution kinetics but this has been shown not to occur for immersion times shorter than 4 hours. On the one hand, it is likely that the slight oxidising character of the pitting agent [8] provokes an iterative cycle of formation/dissolution of a passive layer at the surface of NiAl. Moreover, as discussed previously, fixation of the pitting anion to the polarized organic molecule is also possible hence decreasing its reactivity [19]. Therefore, the stripping solutions should not be excessively concentrated in pitting reagents.

To the best of our knowledge, no published work deals with the dissolution mechanisms of this type of coating in the present experimental conditions and therefore no clear hypothesis can be put forward. The SEM observations of the coated samples stripped for $1 \mathrm{~h}$ in bath A 
can help to understand the likely dissolution mechanisms. It can be noticed in Figure 12 (a) (backscattered electron mode) that the solution seems to penetrate mainly through the grain boundaries of the additive layer of the coating. Then, the solution preferentially attacks the areas with a higher surface energy and therefore reveals the orientation of the (hkl) planes stacking to form the grains. Between the NiAl grains, bright particles appear, most of them embedded in the metallic matrix. These particles are rich in heavy elements (refractory elements) and therefore correspond to the TCP phases and/or carbides [Figure 12(b)]. This phenomenon might be similar in the interdiffusion layer $\left(\mathrm{NiAl} / \mathrm{Ni}_{3} \mathrm{Al}\right.$ matrix), where the solution seems to attack neither the carbides nor the TCP phases (white precipitates). Once stripping has completely dissolved the whole coating, the $\gamma / \gamma^{\prime}$ microstructure of the superalloy is revealed [Figure 12(c)]. Therefore, it seems that the stripping solutions do not fully dissolve the phases. Instead, preferential dissolution of the grain boundaries would occur bringing about detachment of the grains. This interpretation would therefore suggest that stirring of the stripping solutions would be recommended in order to further accelerate the attack.

This mechanism is also verified in the case of the oxidised coatings as shown in Figure 12(d) for a coated (Al-rich) and oxidised sample stripped 4 hours in solution C (Pitting acidOxidising acid-Organic media). It can be observed that some areas still keep the oxide scale that had grown on the original $\mathrm{NiAl}$ grains. Underneath them, the NiAl grains still remain, hence suggesting that the solution has penetrated through the grain boundaries. Between the grains, the interdiffusion layer/substrate interface also shows well defined grains as the attack has proceeded along the grain boundaries. This hypothesis could also explain the difference of behaviour as a function of the initial surface state. Indeed, in the Al-rich coated and oxidised samples many spalled areas appear, which let a fresh coating area appear. Such areas could be preferential sites for solution attack, thus inducing detachment of the grains of the coating by preferential dissolution of grain boundaries instead of dissolving the oxide scale. This hypothesis may be also valid for the Al-poor samples even if they showed lower dissolution rates. In fact, the presence of an oxide overlay (mainly $\mathrm{NiAl}_{2} \mathrm{O}_{4}$ ) at the grain boundaries could hinder the penetration of solutions. Therefore, blasting of the surfaces to remove the corrosion product layers could play a beneficial role in effective stripping of components after service. 


\section{5.- Summary and conclusions}

Various stripping tests have been carried out using different chemical baths at laboratory scale. Stripping has been studied on raw samples, on different CVD aluminide coating qualities and on cyclically oxidised coated samples to approach more actual conditions.

All the baths operated at $50^{\circ} \mathrm{C}$ and stirring for immersion times of $0.5,1,2$ and $4 \mathrm{~h}$. The thermodynamic calculations had indicated that the dissolution reactions were spontaneous either with pitting or oxidising reagents. However, it has been shown that the most adequate reagents are based on a mixture of pitting acid and agent in organic media rather than using oxidising acids because of likely passivation of the surfaces. The aqueous baths are more reactive than the organic ones therefore leading to pitting of the substrates. The most appropriate immersion times to dissolve the coatings while avoiding significant mass loss from the substrate are found between 2 to 4 hours regardless of the bath composition. The results indicate that the stripping mechanisms are governed by specific dissolution of the coatings grain boundaries and/or of the TCP and carbide/metallic interfaces. The same mechanisms would apply for the oxidised specimens as far as the solution penetrates through a defective oxide scale. Therefore, blasting operations prior to chemical stripping are highly recommended.

\section{6.- Acknowledgements}

This work was partly funded by SIFCO Turbine Components, who also supplied the uncoated and the aluminised specimens. The authors are greatly indebted for their help and support.

\section{7.- References}

1. J.R. Nicholls, MRS Bulletin, 28, No.9, (2003) 659-670.

2. M. Eskner, Mechanical properties of gas turbine coatings, Doctoral Thesis. Royal Institute of Technology, Stockholm, (2004).

3. N.A. Amirkhanova, R.R. Nev'yantseva, Protection of metals, 39, No.5, (2003) 538-541.

4. EU Directive 2006/11/CE on pollution caused by certain dangerous substances discharged into the aquatic environment of the Community.

5. L. S. MacDonald, D. Sangeeta, M. A. Rosenzweig, Method for removing an aluminide coating from a substrate, Patent number EP1050604, (2000).

6. J.H. Bowden Jr, J. A. Conner, M.A Rosenzweig, Method for removing high temperature corrosion product from diffusion aluminide coating, Patent number EP1013797, (2000). 
7. SIFCO Turbine Components, CFM-5a HPT blade ST-1300 Lab strip evaluation (Internal communication, 2004).

8. J-F. Martinez, Stripping of aluminide coatings and oxide products on $\mathrm{Ni}$ - based superalloys. Technical Engineer Report. SIFCO Turbine Components - University of La Rochelle (2004).

9. A.Roine, HSC Chemistry for windows Version 3.02, Outokumpu Research Oy, Pori, Finland (2004).

10. F. Pedraza, Effects of ion implantation of silicon, molybdenum and cerium on the high temperature corrosion resistance of different structural steels, Master's Thesis. Universidad Complutense de Madrid (1997).

11. J.H.Chen, J.A. Little, Surf. Coatings Technol. 92 (1997) 69-77.

12. P Castello, F.H. Stott, F. Gesmundo, Corr. Sci. 41 (1999) 901-918.

13. B.M. Warnes, N.S. DuShane, J.E. Cockerill, Surf. Coatings Technol. 148 (2001) 163-170.

14. H. Svensson, J. Angenete, K. Stiller, Surf. Coatings Technol. 177-178 (2004) 152-157.

15. A. Littner, M. Schütze, The cyclic oxidation behaviour of several aluminide and platinum diffusion coatings at $1150^{\circ} \mathrm{C}$, Proceedings of the Corrosion Science in the $21^{\text {st }}$ Century Congress, Manchester (GB), 6-11 July 2003.

16. F.J. Pérez, M.P. Hierro, F. Pedraza, M.C. Carpintero, C. Gómez, R. Tarín, Surf. Coatings Technol. 145 (2001), 1-7.

17. A. Littner, F. Pedraza, A.D. Kennedy, P. Moretto, L. Peichl, T. Weber, M. Schuetze, Mat. High Temp. 22 (2005) 411-420.

18. F.J. Pérez, F. Pedraza, M.P. Hierro, J. Balmain, G. Bonnet, Oxid. Met. 58 (5/6) (2002), 563-588.

19. F. \& A. Rouessac, Analyse chimique $6{ }^{e}$ édition, Ed. Dunod, Paris, 2004.

20. A.M.Shams El Din, F.M. Al-Kharafi, Z. Al-Fahd, Y.A. El-Tantawy, Corrosion Prevention and Control 32 (1985) 92-98.

21. V.B. Singh, U. Arvind, Mat. Corr 46 (1995) 590-594.

22. M.W. Chen, M.L. Glynn, R.T. Ott, T.C. Hufnagel, K.J. Heuker, Acta Mater. 51 (2003) 4279-4294. 

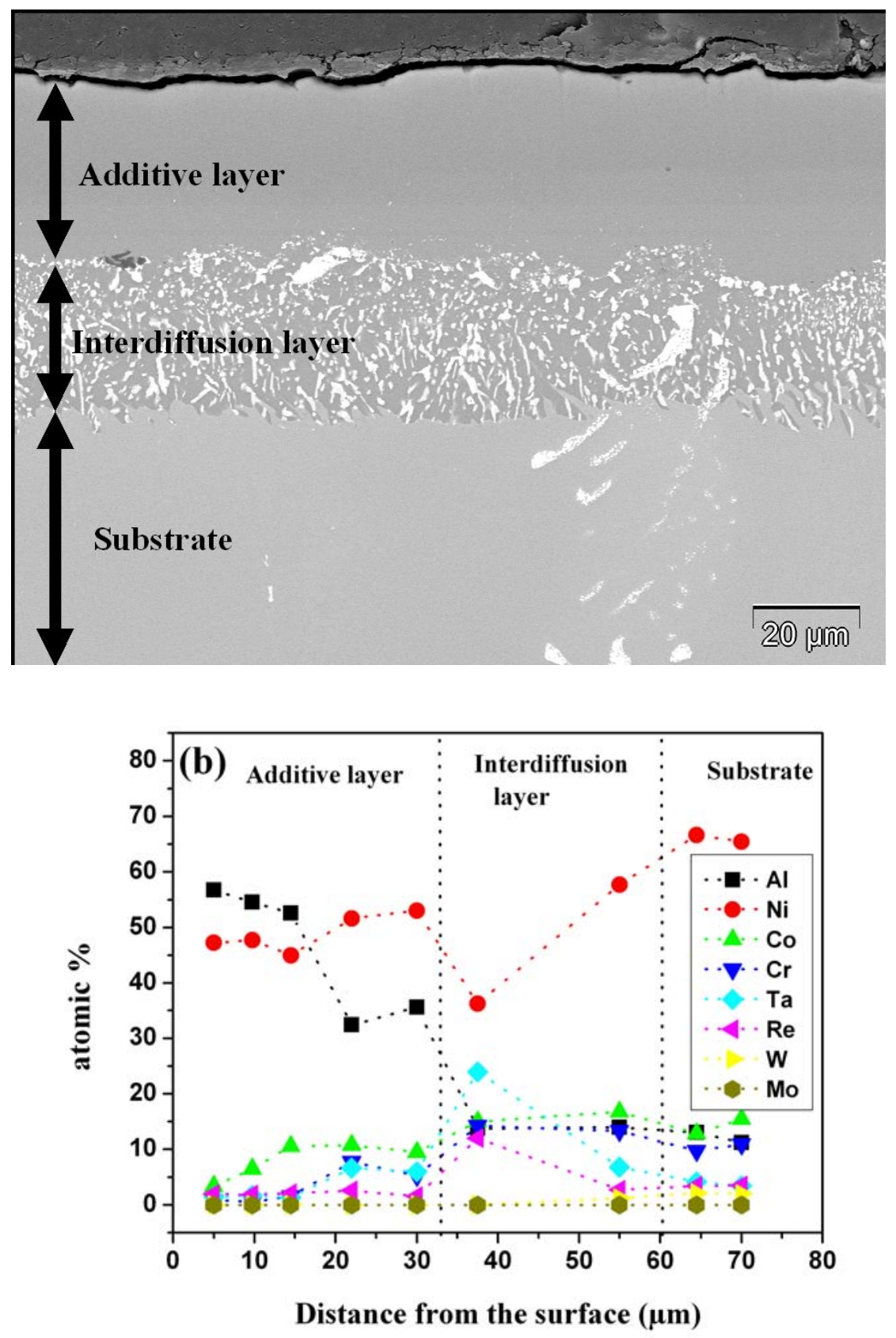

Figure 1.- (a) SEM cross section morphology and (b) EDS microanalyses across the aluminide coating. 


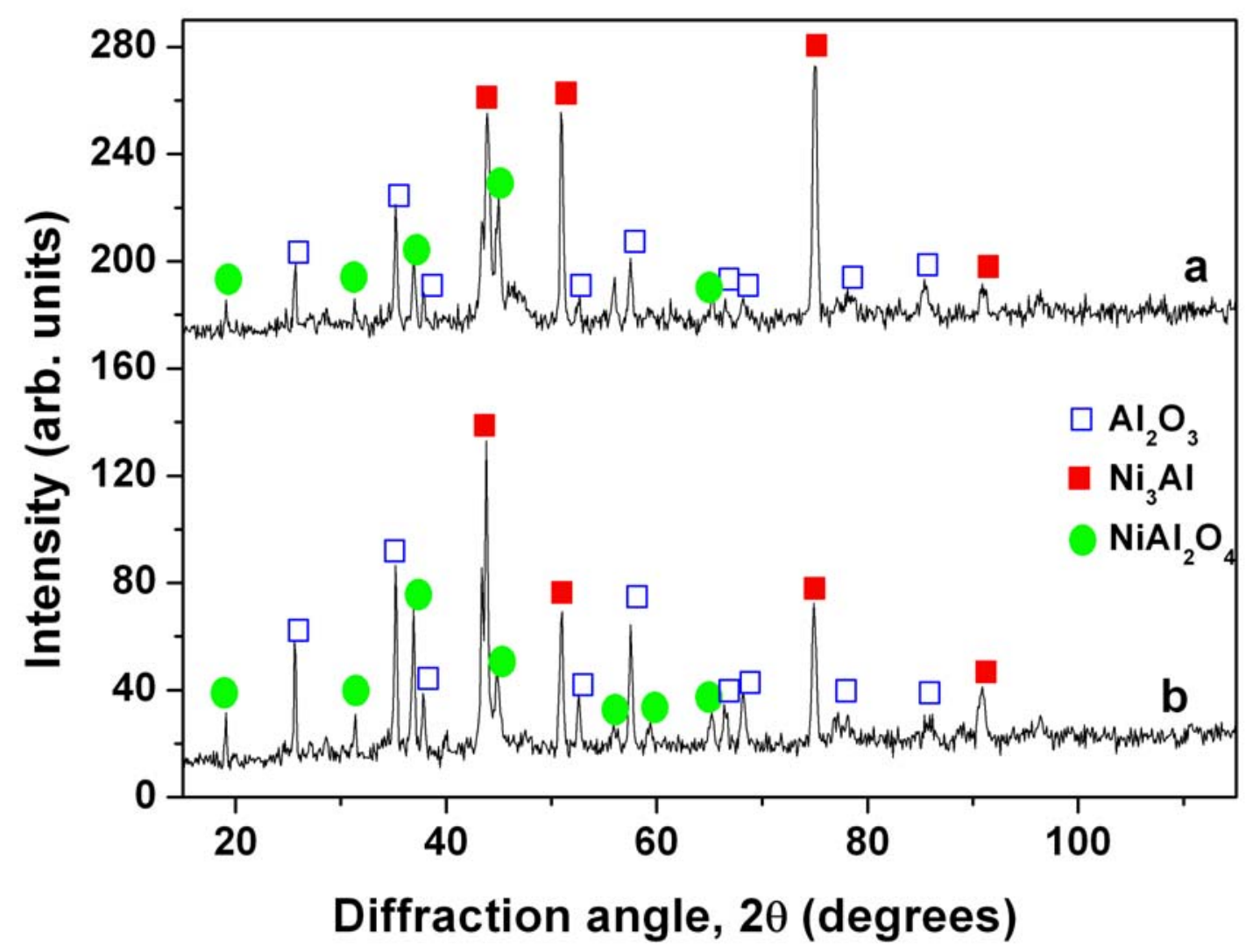

Figure 2.- XRD pattern of coated substrate after $240 \mathrm{~h}$ cyclic oxidation at $1100^{\circ} \mathrm{C}$ in air. (a) Al-poor coating and (b) Al-rich coating. 

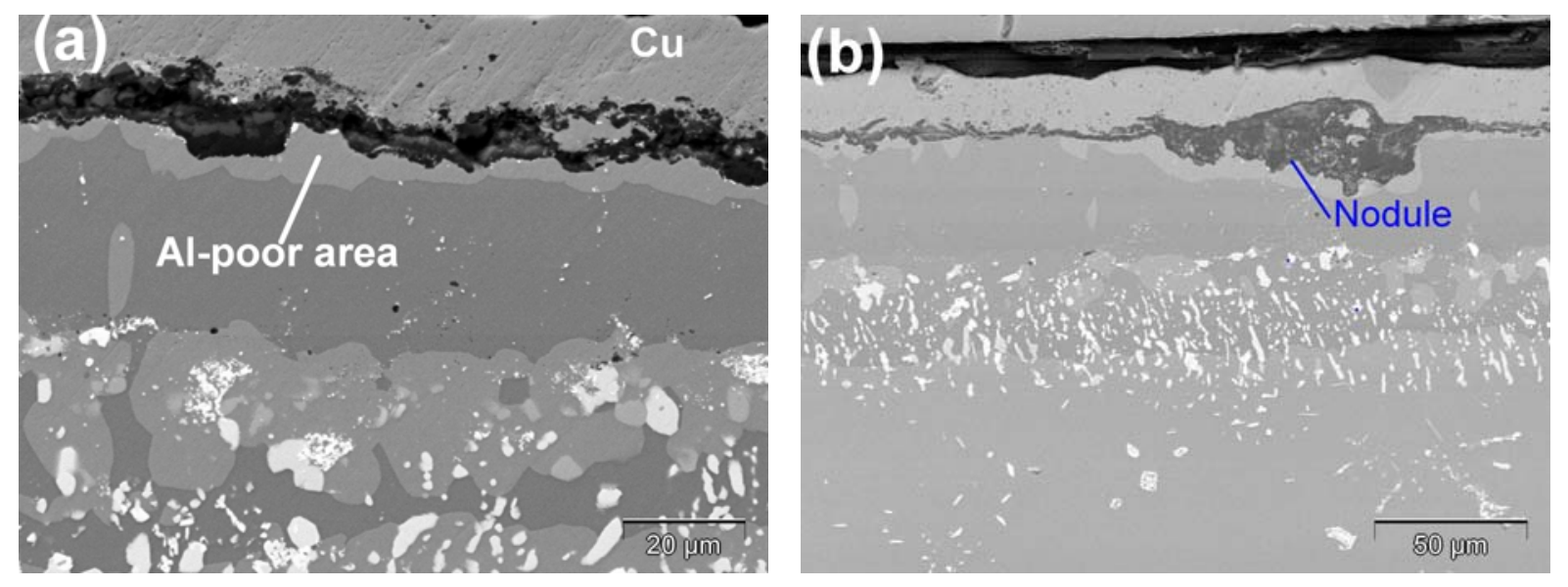

Figure 3.- SEM morphology (BSE mode) of samples surface after $240 \mathrm{~h}$ cyclic oxidation (10 cycles) at $1100^{\circ} \mathrm{C}$ of (a) Al-poor and (b) Al-rich coatings. 


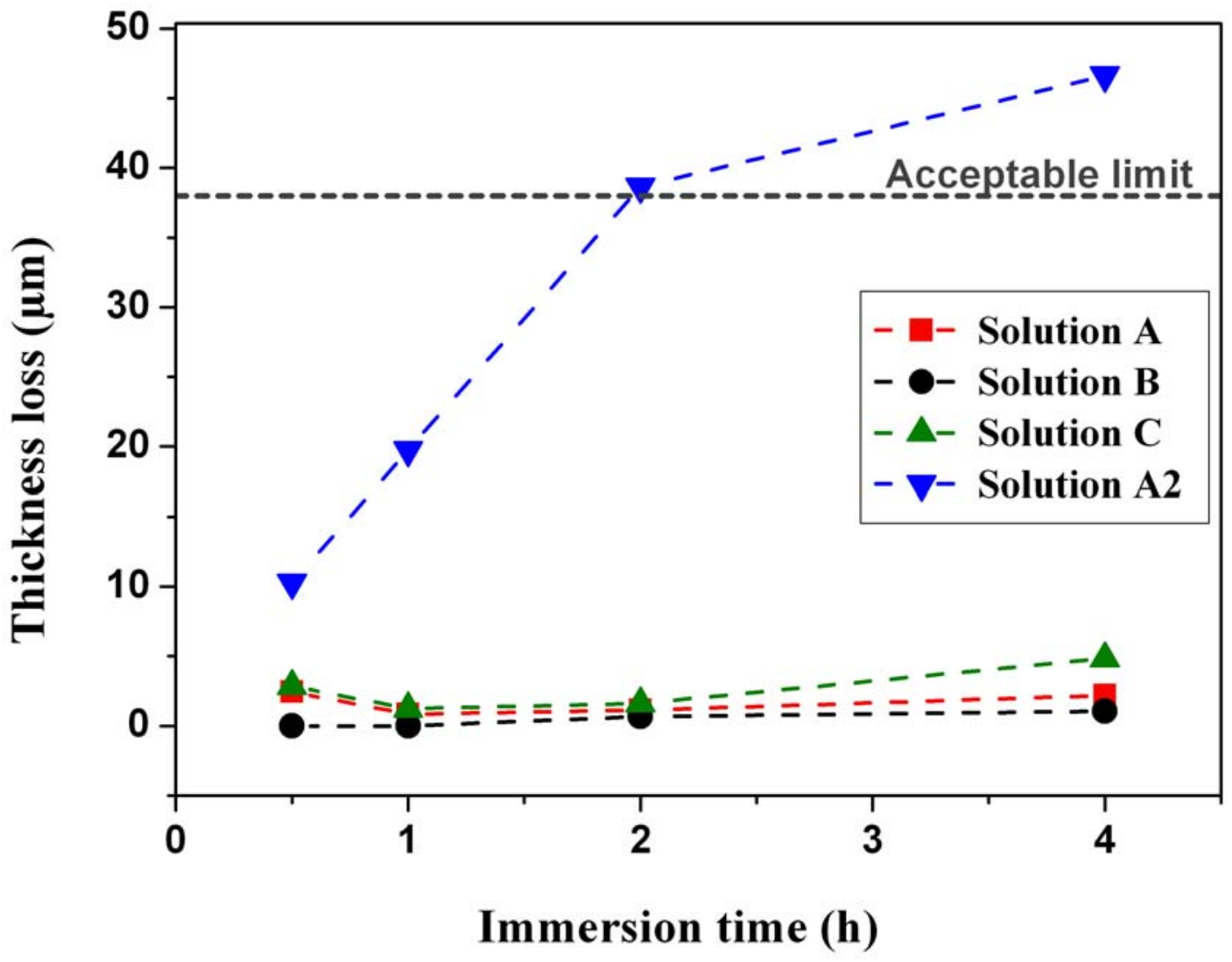

Figure 4.- Estimation of the mass loss with immersion time of the raw superalloy substrate in the different stripping solutions. 


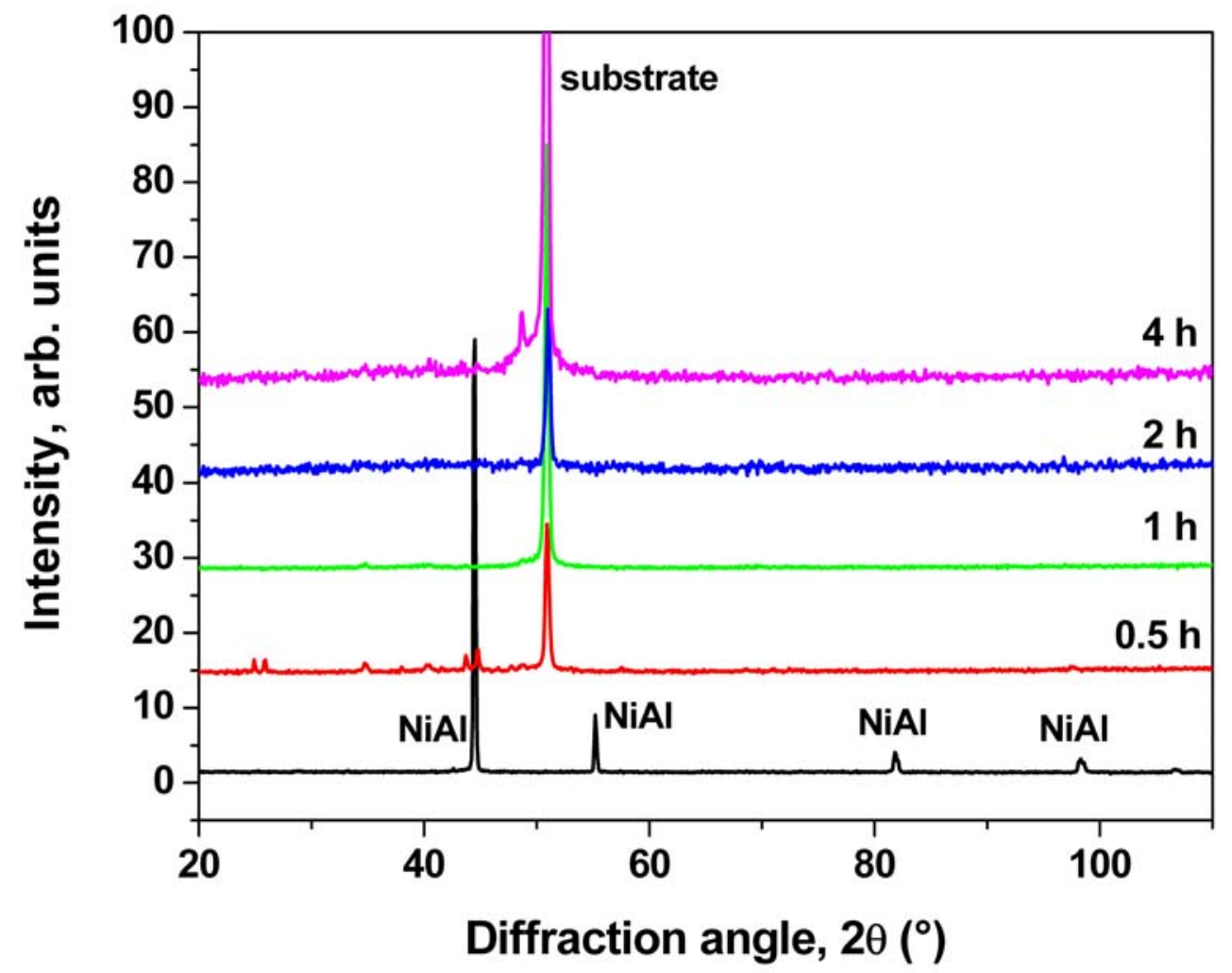

Figure 5.- XRD patterns of the aluminised superalloy samples as a function of stripping immersion time in bath A (Pitting acid- Organic media) at $\mathrm{T}=50^{\circ} \mathrm{C}$. 

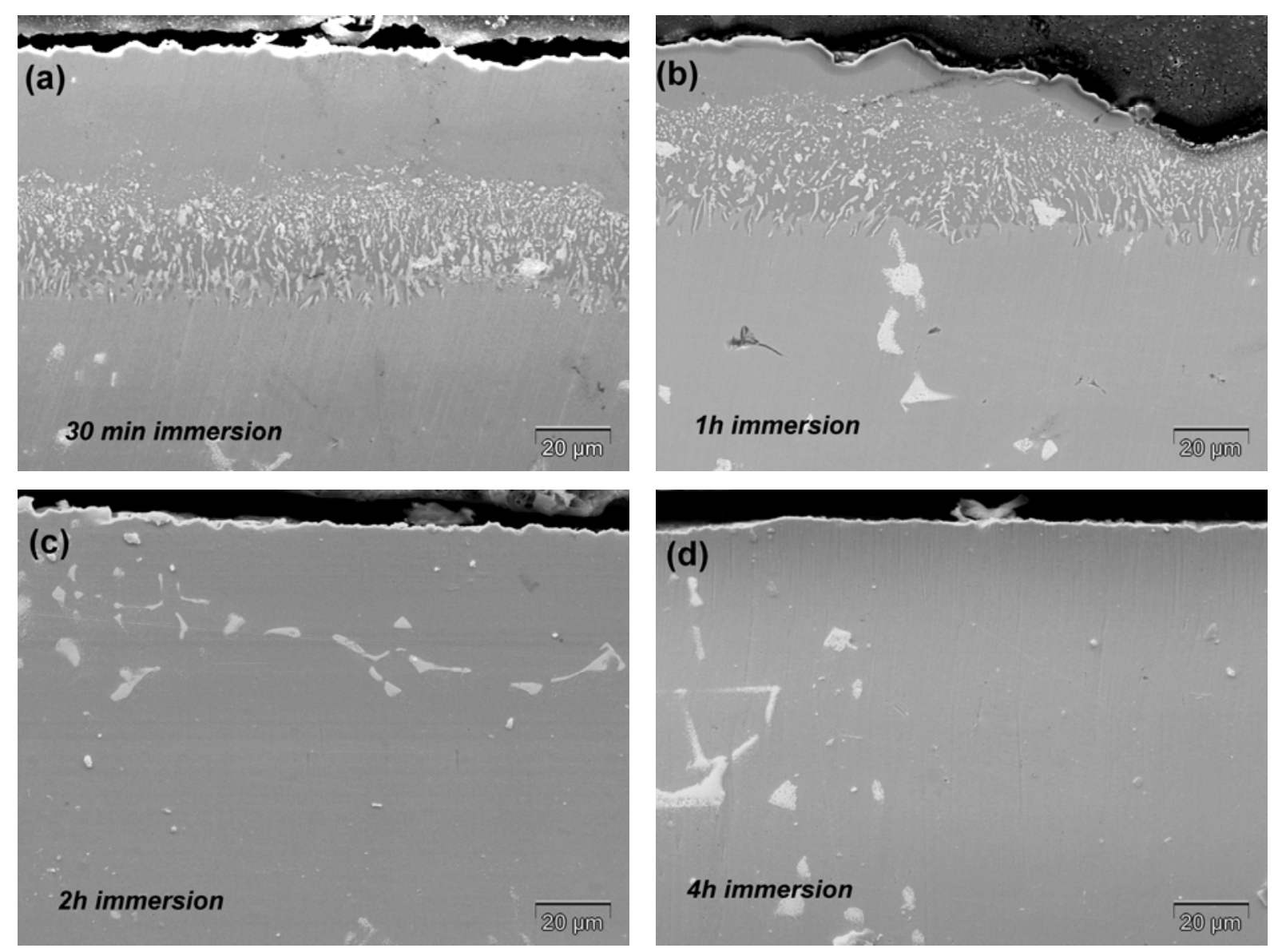

Figure 6.- Evolution of the cross section morphology of the aluminised substrate with stripping time in bath A (Pitting acid- Organic media). 


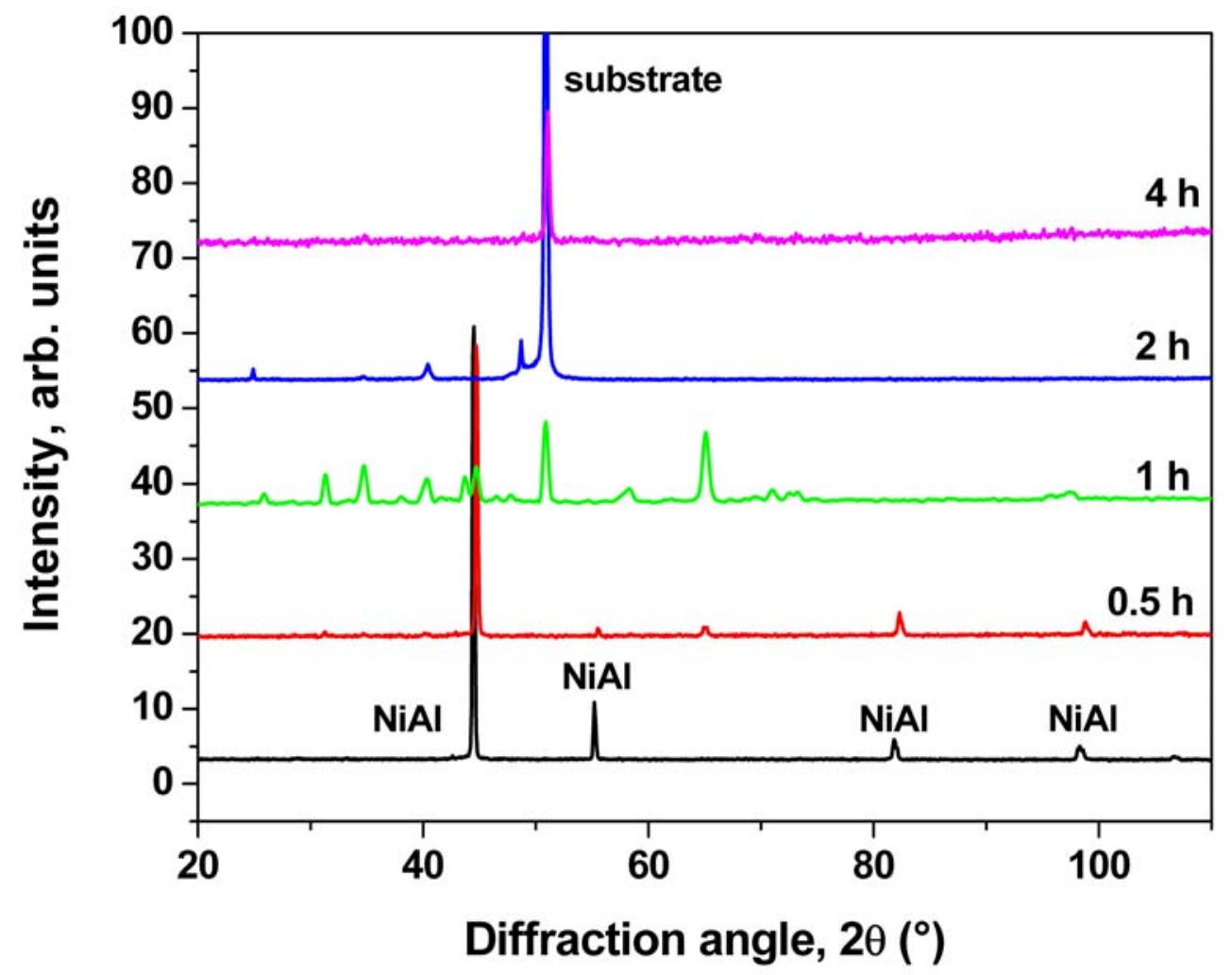

Figure 7.- XRD patterns of the aluminised samples as a function of stripping immersion time in bath $\mathrm{C}$ (Pitting acid-Oxidising acid-Organic media) at $\mathrm{T}=50^{\circ} \mathrm{C}$. 


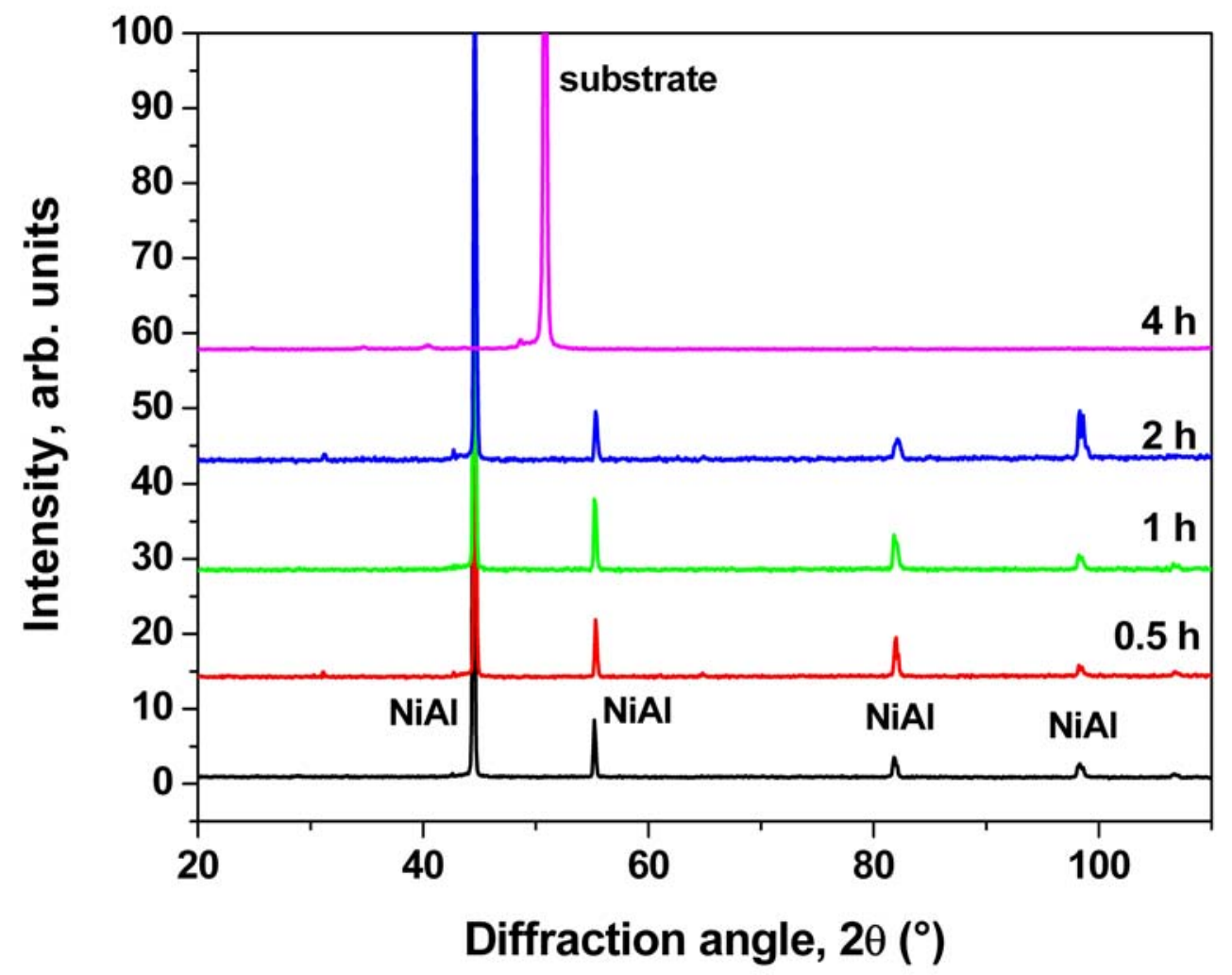

Figure 8.- XRD patterns of the aluminised DSR142 samples as a function of stripping immersion time in bath A2 (Pitting acid-Pitting agent-Organic media) at $\mathrm{T}=50^{\circ} \mathrm{C}$. 

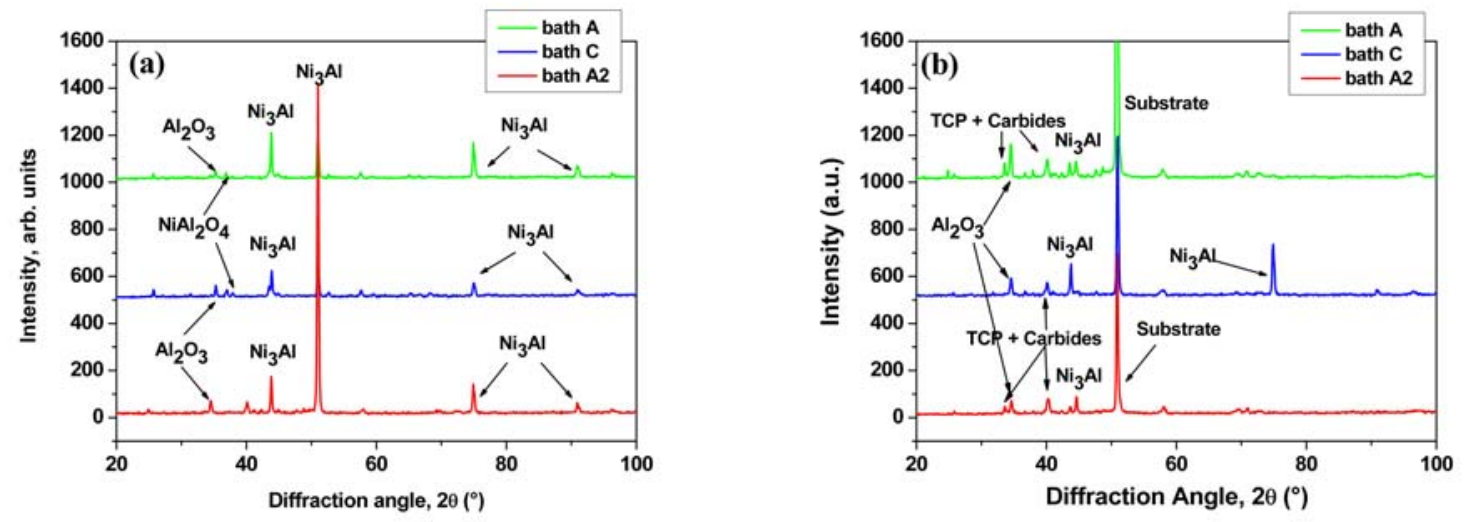

Figure 9.- XRD patterns of the samples after 4 hours stripping of (a) Al-poor and (b) Al-rich coatings after cyclic oxidation at $1100^{\circ} \mathrm{C}$ for $240 \mathrm{~h}$. 

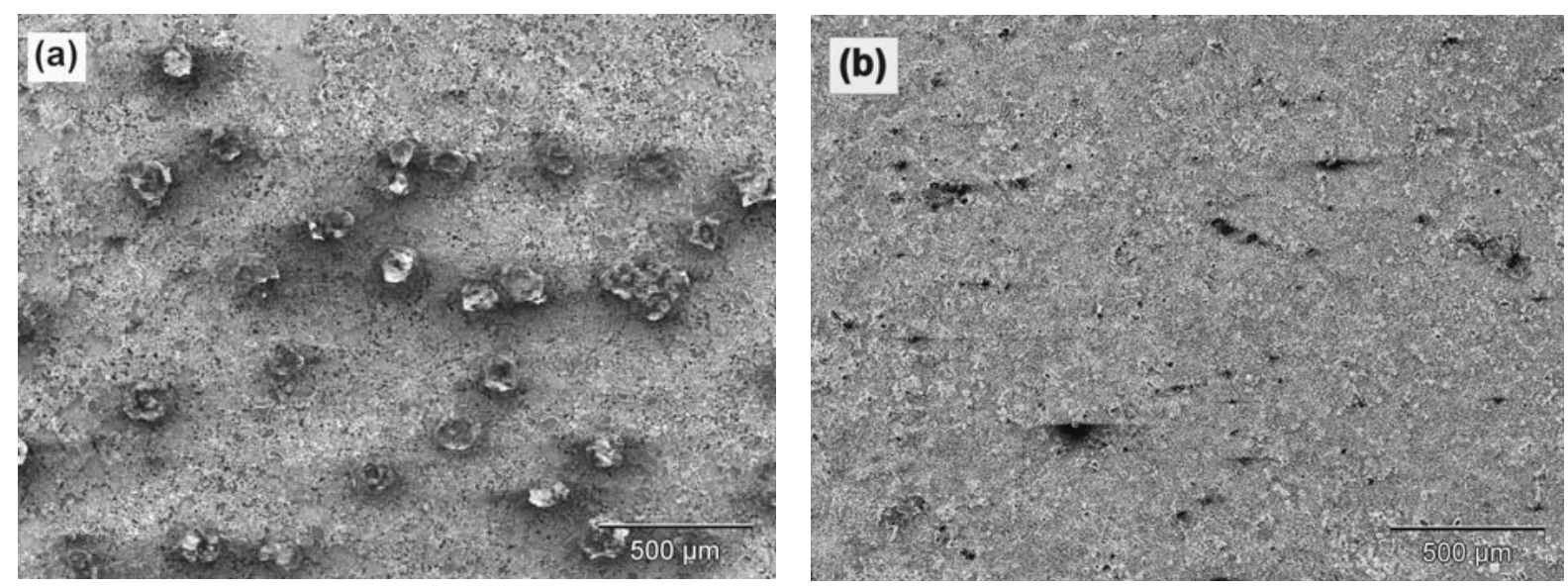

Figure 10.- SEM surfaces after 4 hours of stripping in baths (a) A, (b) C and (c) A2 of Al-rich coatings after $240 \mathrm{~h}$ cyclic oxidation at $1100^{\circ} \mathrm{C}$. 


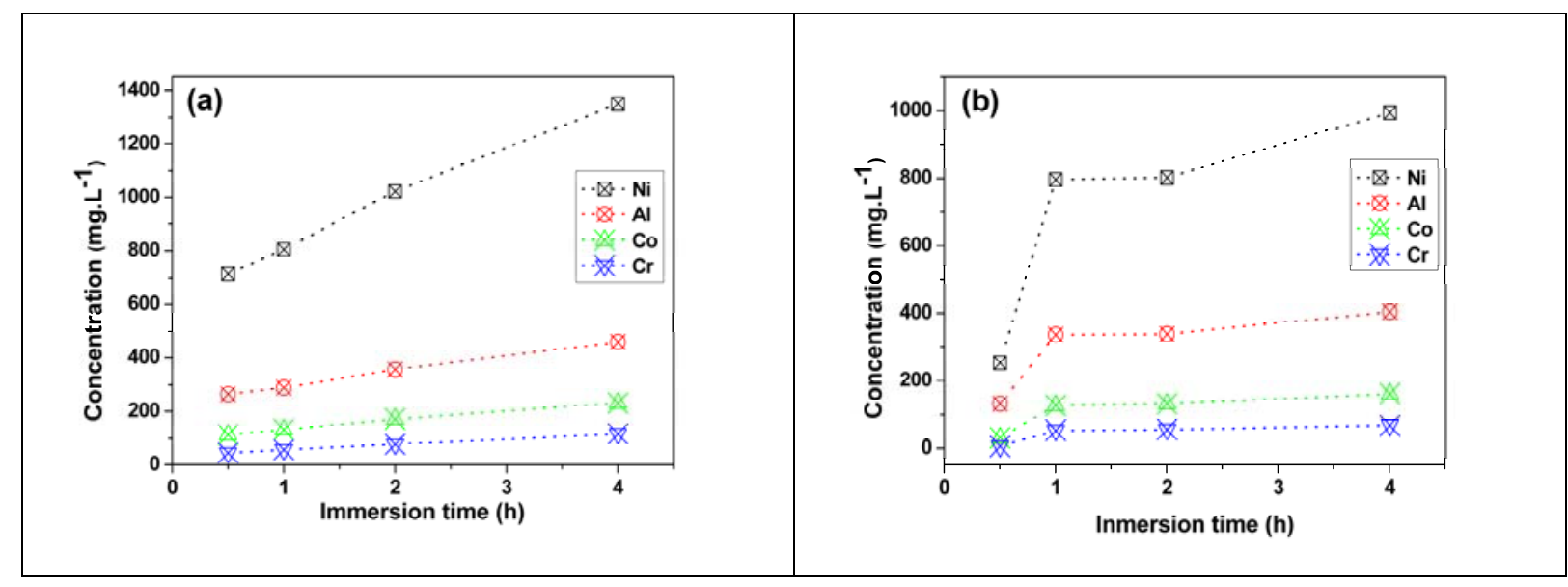

Figure 11.- Evolution of the concentration of the major elements in solution after stripping in baths (a) A and (b) C obtained by ICP-OES. 

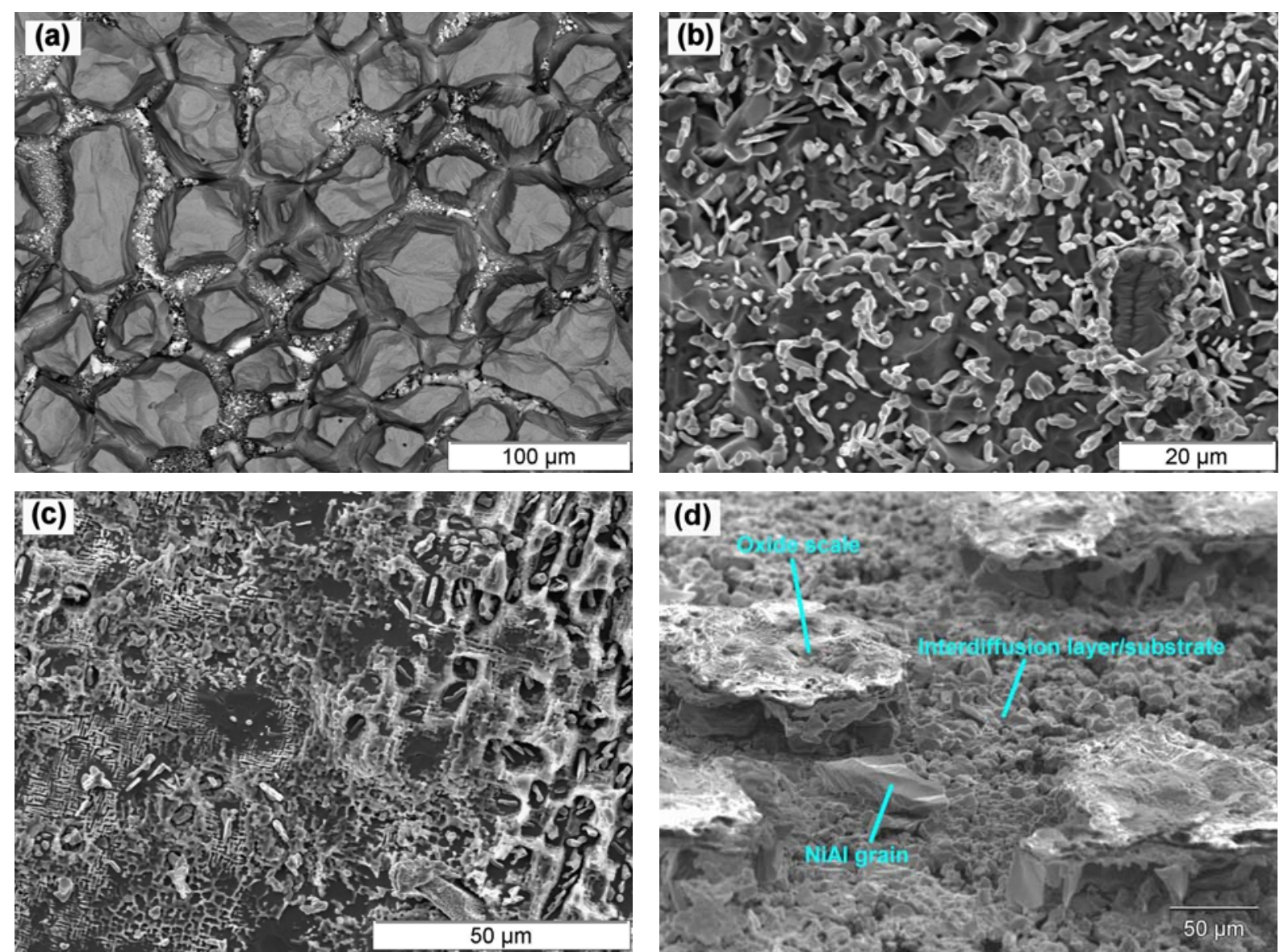

Figure 12.- Evolution of the dissolution of the as-coated specimens with immersion time in bath A (a) $30 \mathrm{~min}$, (b) $1 \mathrm{~h}$ and (c) $4 \mathrm{~h}$. Stripping of an oxidised Al-rich coating with bath $\mathrm{C}$ for $4 \mathrm{~h}$ specimen tilted at $60^{\circ}$ ). 